\title{
7
}

\section{Prebiotics and Dietary Fibers from Food Processing By-Products}

\author{
Santad Wichienchot ${ }^{1, *}$ and Wan Rosli Bin Wan Ishak ${ }^{2}$ \\ ${ }^{1}$ Prince of Songkla University, Hat Yai, Songkhla, Thailand \\ ${ }^{2}$ Universiti Sains Malaysia Health Campus, Kubang Kerian, Kota Bharu, Kelantan, Malaysia
}

\subsection{Introduction}

The abundance of agricultural wastes or by-products from industrial and domesticated food processing is the main cause of environment problems. These by-products are generally managed by disposal or even sold at a cheaper price. Disposal of these underutilized by-products are commonly done in inappropriate ways, i.e. discharge effluent into rivers or by burning in the open, which may cause air and water pollutions. Presently, scientific investigation on the benefits or functional properties of waste and by-products from industrial food processing, which produces a large amount of by-products, is necessary in the search for possible ways for their utilization (Vanesa et al., 2011). Three main groups of by-product from food processing, classified according to their main chemical compositions, are carbohydrate and dietary fibers, protein and lipids. The most common by-products are generated by the food industry, in particular the beverage, starch and flour industries. These items are classified under carbohydrate and dietary fiber groups. They are further divided into four sub-groups: monosaccharides, disaccharides, oligosaccharides and polysaccharides.

Dietary fibers are a class of non-starch polysaccharides (i.e. cellulose, dextrins, chitins, pectins, $\beta$-glucans and waxes) and lignin, which are able to modulate the transit time through the gut. Thus, it provides similar beneficial effects to those of inulin-type fructans. These compounds are commonly found in many foods such as cereal, nuts etc. They are also partially susceptible to bacterial fermentation and may induce changes in bacterial populations, particularly in the numerous bifidobacteria and lactobacilli. These soluble dietary fibers have been shown to exert additional beneficial effects, for instance by improving gut barrier function in vitro and in vivo, which could be partially a consequence of their effect on the microflora composition (Laparra and Sanz, 2010).

Food Processing By-Products and their Utilization, First Edition. Edited by Anil K Anal. (C) 2017 John Wiley \& Sons Ltd. Published 2017 by John Wiley \& Sons Ltd. 